\title{
The Biological Pigments in Plants Physiology
}

\author{
Arturo Solís Herrera \\ Human Photosynthesis ${ }^{\circledR}$ Study Center, Aguascalientes, Mexico \\ Email: comagua2000@yahoo.com
}

Received 7 October 2015; accepted 26 October 2015; published 29 October 2015

Copyright (C) 2015 by author and Scientific Research Publishing Inc.

This work is licensed under the Creative Commons Attribution International License (CC BY).

http://creativecommons.org/licenses/by/4.0/

(c) (i) Open Access

\begin{abstract}
The physiology of the plants has come to be a formidable challenge. Despite best efforts, the mysteries remain, most of the processes identified cannot be played in vitro, and to date it cannot be said that knowledge about the complex biochemical processes of the plant is understood properly. The need to produce food is an emergency in the world, so any knowledge that will allow us to advance in this sense is important. Our discovery about the intrinsic property of melanin transform light energy into chemical energy by means of the dissociation of the molecule of water, such as chlorophyll in plants, represents a turning point in relation to the chemical reaction so far considered the most important in the world: photosynthesis.
\end{abstract}

\section{Keywords}

\section{Chlorophyll, Melanin, Lignin, Water Dissociation, Hydrogen, Oxygen, Energy, Pigments}

\section{Introduction}

A pigment is defined as any substance capable of absorbing light [1], so it could be considered pigments almost all substances. Usually, substances absorb very specific wavelengths, and emit a characteristic electromagnetic signal that allows us to identify them. Color is the property of electromagnetic radiation with a wavelength between 300 - 400 and $700-800 \mathrm{~nm}$. The color of individual chemical substances derived from their interaction with white light. If the total radiation falling on a chemical compound passes through or is reflected from it, the substance has a white color. If white light on contact with a substance is completely absorbed, appears black. Most often chemical compounds selectively absorb within certain radiation ranges and the color of a given compound is complementary to the absorbed radiation.

This allows us to infer that the energy inherent in the light produced a change in substance or molecule that it absorbed, so far in accordance with the law of conservation of energy, whose first postulate says that energy, 
defined as everything that produces a change is not created or destroyed, only is thus transformed.

Therefore, the light energy absorbed by the compound or molecule, brought about a change in it, and the new molecule issued part of the absorbed energy, in the form of electromagnetic signal, for example, light with different wavelength to which was originally absorbed.

Nature is full of examples in this regard, and the plants are no exception. And also in them it happens that the wavelengths of light can be, wholly or partly, absorbed, transmitted or reflected.

And many compounds containing a plant that they can classified as pigments, for example: porphyrins, carotenoids, anthocyanins, etc. However, there are two that have powerfully drawn attention: the chlorophyll and recently the melanin. And the reason is that both molecules have very peculiar behaviors, we could call them unique in nature. This is: both molecules are able to dissociate the water molecule.

And it is the attention, because we are against the way in which nature transforms light energy into chemical energy. It is possible that there are other molecules that can do so, but the two examples most known or at least shown are chlorophyll, which until a decade ago was considered the only molecule with such capacity, and recently, melanin.

Chlorophyll, absorbs the ends of the visible spectrum, and with that power separates the liquid water in its gaseous components, hydrogen and molecular oxygen; and it carried out at room temperature and in an irreversible manner, then ejects the oxygen into the atmosphere. The chemical reaction can be written in the following way.

$$
2 \mathrm{H}_{2} \mathrm{O}_{\text {(liquid) }} \rightarrow 2 \mathrm{H}_{2 \text { (gas) }}+\mathrm{O}_{2 \text { (gas) }}
$$

It is the attention that the reaction happens so quickly and at room temperature inside the chlorophyll's leaves, as in the laboratory we can dissociate water, but requires warm water at $2000^{\circ} \mathrm{C}$ and the products that we get, they are not so ordered as in chlorophyll and melanin. This reaction is impelled by the extremes of visible light (violet and red)

Another interesting detail is that part of the energy that is released when water dissociates, is transported by molecular hydrogen, which was to be expected since it is the main carrier of energy in the entire universe.

On the other hand, molecular oxygen, which is very stable and toxic, is expelled into the atmosphere by the plant, and since it has always been present in the equation, the plant optimizes it, given its high toxicity.

In melanin (Greek: melanos; dark), the reaction is similar, but quicker and thousands of times more efficient, because the melanin absorbs electromagnetic full spectrum and in addition is capable not only of separate water into its components but also re-shape the molecule, this is: gaseous components takes her to the liquid state again [2]. This reaction happens inside melanin, and can be located both intra and extracellularly. The chemical reaction is written thus:

$$
2 \mathrm{H}_{2} \mathrm{O}_{\text {(liquid) }} \rightarrow 2 \mathrm{H}_{2 \text { (gas) }}+\mathrm{O}_{2 \text { (gas) }} \rightarrow 2 \mathrm{H}_{2} \mathrm{O}_{\text {(liquid) }}+4 \mathrm{e}^{-}
$$

Or so:

$$
2 \mathrm{H}_{2} \mathrm{O}_{\text {(liquid) }} \leftrightarrow 2 \mathrm{H}_{2 \text { (gas) }}+\mathrm{O}_{2 \text { (gas) }}+4 \mathrm{e}^{-}
$$

For every two molecules of water re-formed, are generated 4 high energy electrons, which are electrons whose probability cloud is located farthest from the atomic nucleus in question as a low-energy electron or stable state. High energy electrons or excited are exchanged easily, making it almost impossible to identify the atom or molecule to which they belong.

\section{Melanin, the Meta-Chlorophyll}

Melanin is what lies behind the chlorophyll, which explains the misunderstood chlorophyll, which remedied the deficiencies; in few words, is that even explains the origin of life.

Chemicals Processes are known in plants, which the mere dissociation of water by chlorophyll cannot explain, could tell that the physiology of the plant is full of mysteries until now misunderstood. The energy that emanates from the chlorophyll does not provide a satisfactory explanation for so many and so many observed phenomena, both in the root, stem and leaves. Only the circulation of the SAP not has been explained to date.

The so-called cohesion-tension theory that try to explain SAP transport by the xylem, from the roots of the 
plant to the leaves through the wood tubules, is to date; a theory.

The mysterious forces that drive the viscous solutions through the trunk, from the root to the leaves, in order, in a sequence that is not understood, no doubt require energy, cannot be explained by the chlorophyll, glucose or ATP, but the energy that emanates from the melanin comes to fill a hole that will allow us to advance in the understanding of the intricate biochemical processes that affect the life of the plant.

Melanin in humans had passed as unnoticed as the lignin in plants, the main interest at present was to modify the composition of lignin, which is the melanin in the plants; as a basic strategy to increase the digestibility of cell walls in order to produce biodiesel [3].

It is expected that as you go knowing the unsuspected intrinsic property of melanin transform light energy into chemical energy by means of the water molecule dissociation, the interest becomes more reasonable, because the omnipresence of melanin explains the germination of the seeds, as it was a mystery as the seed detected level appropriate water to flourish (Figures 1-3).

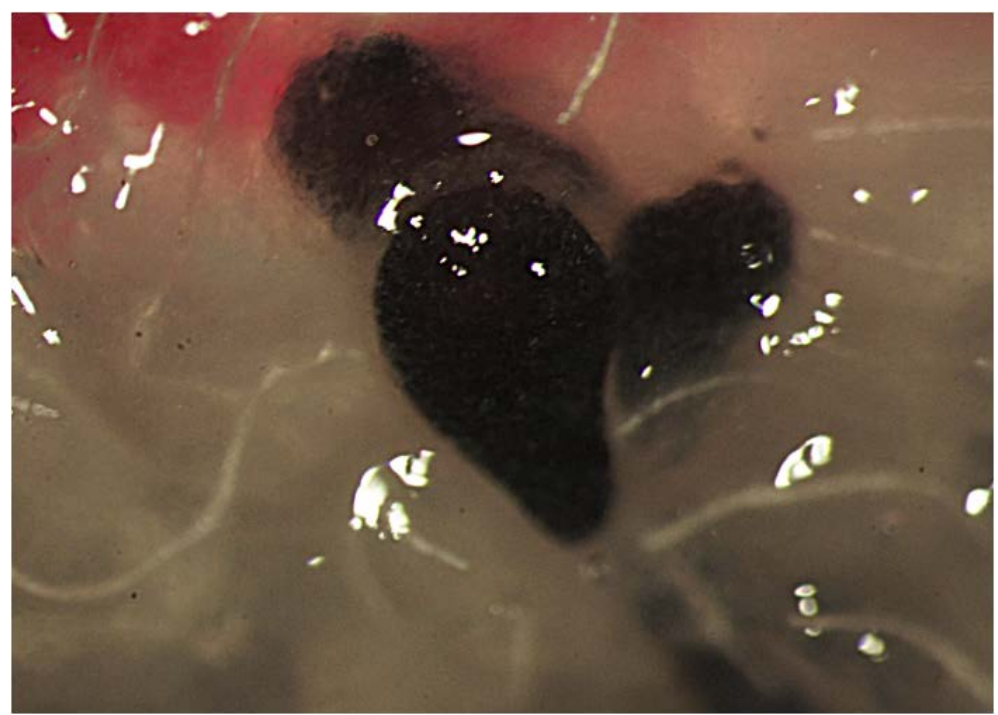

Figure 1. Melanin is present in practically all of the seeds, and the pitaya is no exception. When the amount of water is scarce, power that can produce melanin also is, by what the mechanisms leading to the emergence of seed not can be driven, when the amount of water is adequate, then the energy that emanates from the melanin will be adequate to promote each and every one of the mechanisms that lead to the germination of the seeds $(16 \times)$.

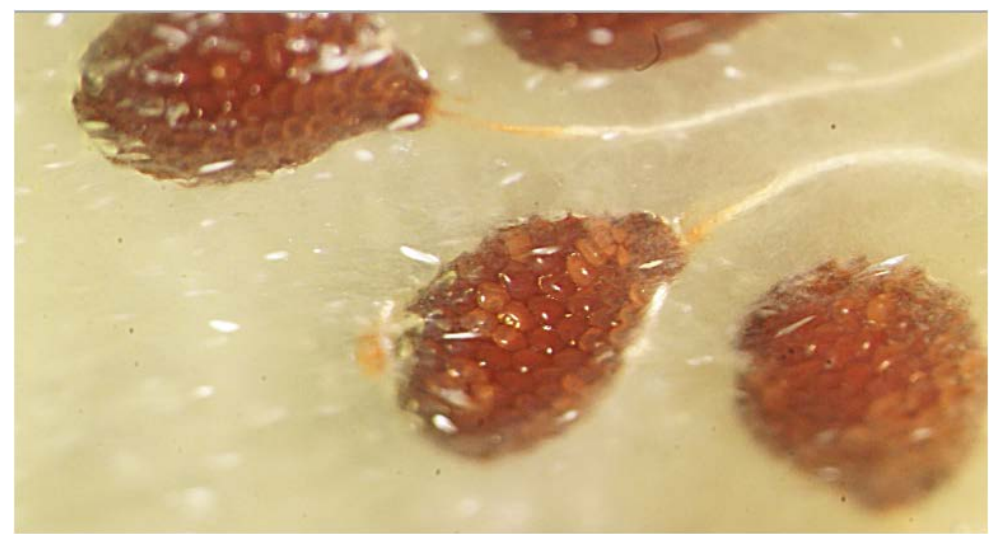

Figure 2. Seed color depends on the amount of melanin, granule size, the orientation of the molecule, the combination with other compounds, as well as the nature of the structures that are in, but, either way, its main function is the same: production of chemical energy $(16 \times)$. 


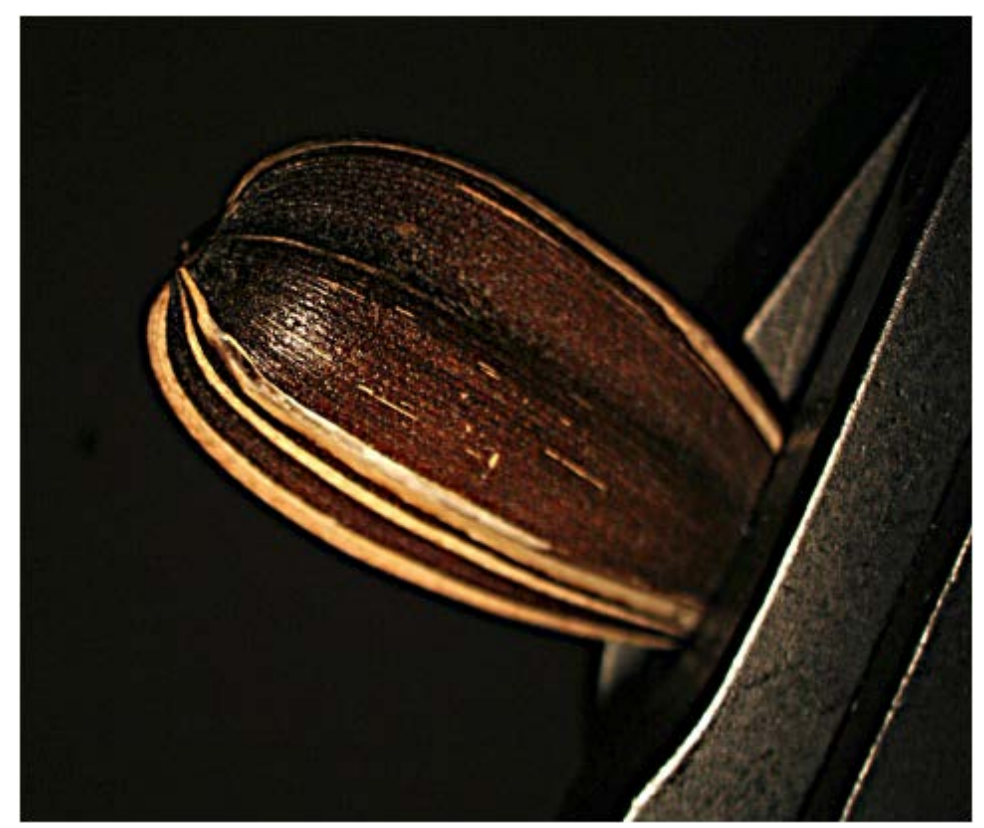

Figure 3. The same principle applies in all living organisms, apparently is the only or at least the best system to convert light energy into chemical energy. In Sunflower seed we can see melanin. The magnification is $25 \times$.

The similarity of the surface of the iris of the human eye (Figure 4, Figure 5) with the surface of some seeds, for example, the peach, are given by the notable content of melanin in both structures.

The concept that photosynthetic membrane is the microscopic powerhouse of our biosphere, now is broken into small pieces.

Molecular hydrogen and high energy electrons coming from the dissociation of water, due to the melanin molecule, constitute the true universal biological engine. The oxidation of water as the basis of molecular biology is more true than ever, but as the melanindone, chlorophyll only is an additional mechanism that optimizes some biochemical processes, as does the hemoglobin in human body, as it is a molecule that also has the amazing ability to dissociate the water molecule irreversibly, and was to be expected, because the biochemical differences between chlorophyll and hemoglobin are really minimal.

Because of the huge differences between melanin and chlorophyll, it is that, based on this last, it has not been possible to explain the metabolism of plants. To date, the questions are many, despite the best efforts of researchers and Governments that support research.

\section{Brief History of the Discovery}

Since melanin absorbs all kinds of energy and dissipates it separating the water molecule, their behavior is unique in nature, because it does not emit any electromagnetic, visible or invisible signal that will allow us to characterize it. As a result, the study of melanin in the laboratory has been the date; a formidable challenge [4]. So it is understandable that the finding of its intrinsic property of transform light energy into chemical energy, by means of the water molecule dissociation, came from another type of study, in this case, of a clinical trial; because we observed incidentally melanin acting in one of its multiple location that is in nature. Why is that we consider important to a brief description of the how it came to knowledge.

In 1990, we started an observational, descriptive, study about the three main causes of blindness in Mexico, and that is no coincidence that is the same around the world: Glaucoma, diabetic retinopathy, and macular degeneration related to age.

Our working hypothesis was trying to characterize the morphology of the blood vessels of the optic nerve, very small structure in humans, whose average diameter is equivalent to twelve human hair together. Our aim was to try to find vascular anatomical changes that could eventually serve as indicators of early disease and therefore allowed us early treatments. 


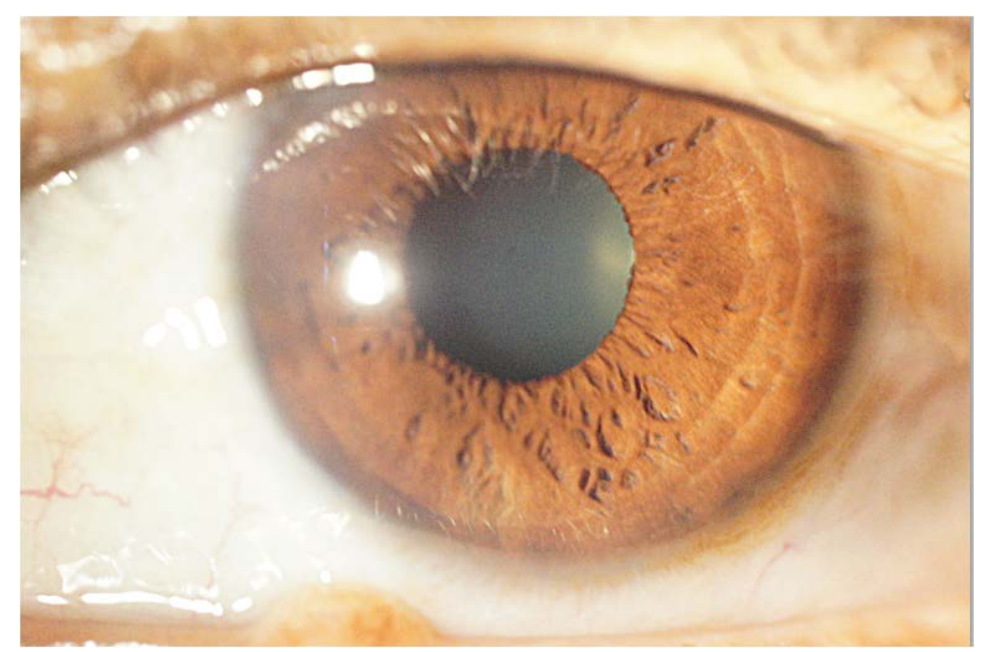

Figure 4. Melanin, wherever you are or whatever that he is called, has the same function, namely the transformation of light, visible and invisible power; in energy chemistry through dissociation and further re-formating of the water molecule. The effect of melanin on the nearest atoms and molecules is surprisingly consistent, as evidenced the huge resemblance between the surface of the iris of the eye and the surface of some seeds, bark of trees, etc.

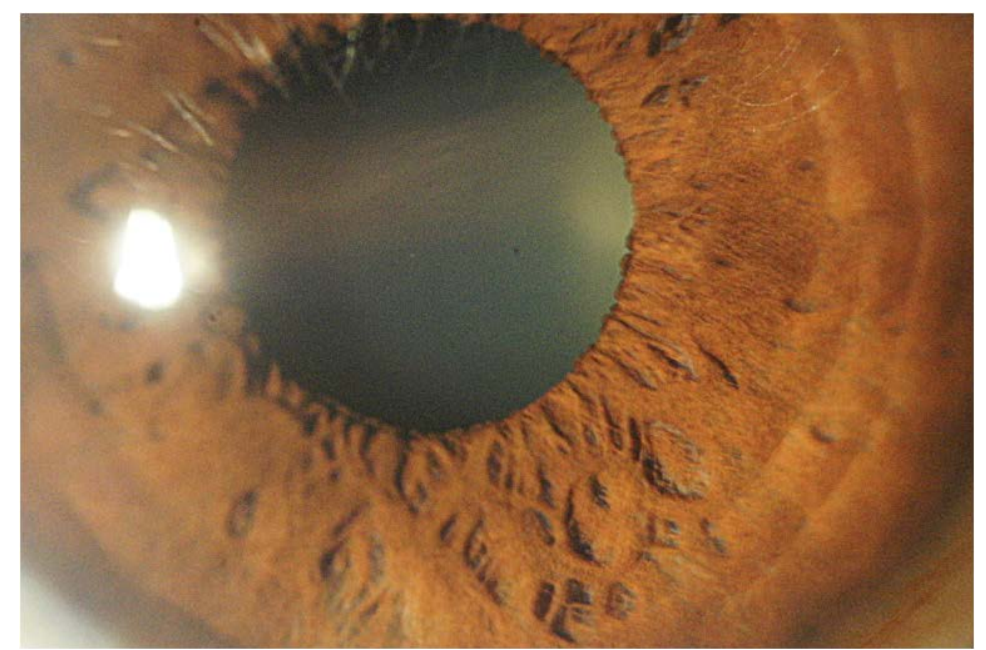

Figure 5. To greater amplification, the resemblance is even greater between the surface of the iris and some seeds, including with the bark of some trees. Is conceivable that at higher magnifications, come a time that would be difficult to discern which is which.

However, the magnifications required to carry out properly the characterization of vascular anatomical pattern, are in the order of $20 \times$ to $40 \times$. And to these amplifications, we started to notice the omnipresence of the melanin in the vicinity of the optic disc (Figure 6).

And the insistence of nature in place melanin in the entirety of the almost 6000 patients who checked in the span of 12 years which lasted the study (1990-2002), struck us mightily, because nature just insists on important things.

So a few months of starting the study, instead of one, there were two main variables in study: blood vessels and melanin.

We were sure that melanin should play an important biological role, but we didn't understand that. Eventually we began to detect an antagonism between the blood vessels and the melanin that was becoming more and more evident. Some time later, we had a conclusion: more melanin, fewer blood vessels and vice versa (Figure 7). 


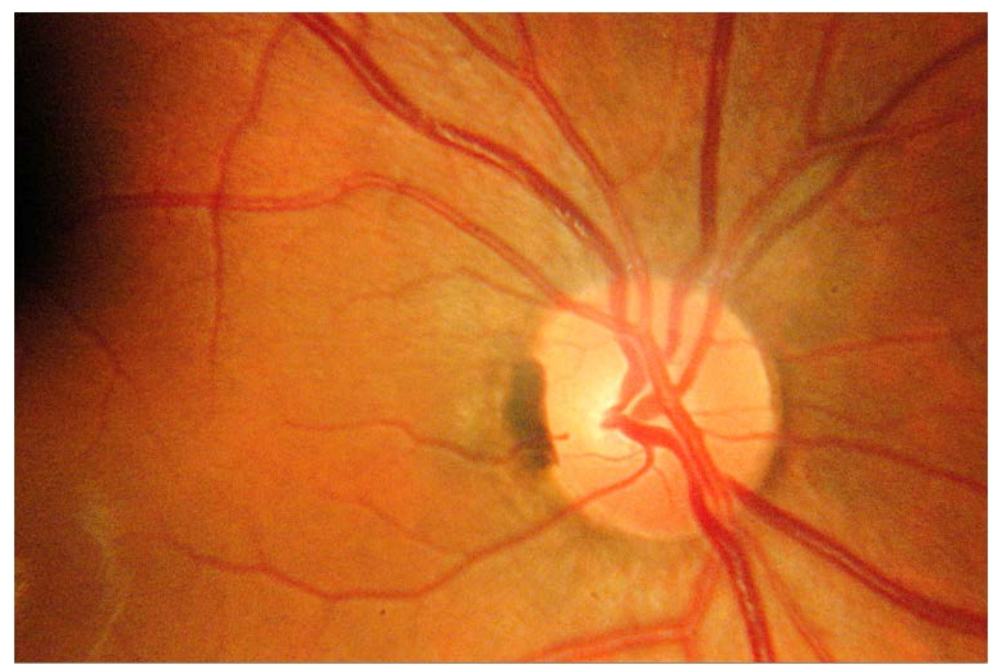

Figure 6. The picture shows the optic nerve of the right eye, with its characteristic orange colour, its oval shape, vessels entering and leaving; and in the meridian of the 9 there is a patch of dark color which corresponds to melanin.

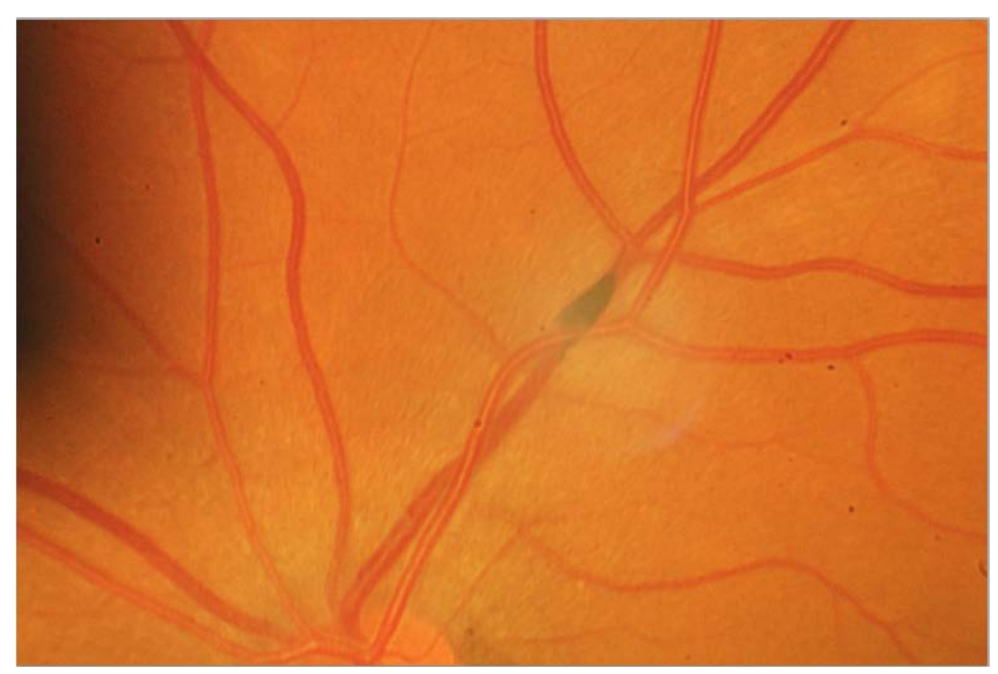

Figure 7. PICTURED are the retinal vessels, and a dark spot that surrounds them partially and which corresponds to the melanin. The effect on the vessels is somewhat subtle, because in this case only observed a light pale around the melanin. In people with fair skin angiogenesis inhibitor effect of melanin is still more subtle, and in dark-skinned people is almost impossible to detect by the amount of melanin.

What remained was to try to explain it. In principle, we did not know which predominated on which. On the one hand, melanin does not possess something like organelles that could produce some kind of transmitters, e.g. peptides, neither nor has something that could be interpreted as receivers. In relation to the blood vessels, these have rough endoplasmic reticulum, so it can synthesize factors that function as transmitters of information, but melanin has no receptors.

And something that was surprising was that the melanin antiangiogenic effect is very powerful, because it is present all the time, which was at odds with some kind of factor, for example: Pigment epithelium derived factor or Vascular endothelial growth factor; because the biological variability in the same factor and the corresponding receiver significantly reduces its effect, perhaps in $60 \%$.

And the effect of melanin is surprisingly consistent, then it had to be something different from the known 
factors. By then, we realized that molecular oxygen levels were different between tissues, according to the amount of pigment. A greater amount of melanin, higher levels of molecular oxygen and vice versa. So the oxygen was the answer, because it is the best anti-angiogenic agent referred to.

We have been solving the unknowns, but now appeared a new: where does oxygen came from? The difference in oxygen levels between the pigmented tissues and the less pigmented, in our laboratory conditions, in the human eye, it goes from $34 \%$ to $54 \%$, a huge difference.

We review several molecules, various tissues, looking for changes consistent with a constant oxygen donation, and did not find anything, it seemed that not passing anything, but a molecule or tissue that had to submit so much oxygen, , sooner or later, will has any perceptible change, and nothing.

Finally, we had only one option: the water containing the eye and that is something more than $95 \%$ of the body. And it was the perfect candidate, because in water is hardly notice the changes. But the water not releasing oxygen for free, have to pay it, i.e. energy is required.

So that left us with an unknown: where does the energy so the water yield their oxygen atoms? And after several unsuccessful attempts, the answer appeared in front of a Blackboard full of cluttered biochemical equations, was February 2002; "He is dissociating water" I thought, and to write the equation on the Blackboard, suddenly appeared the order in the rest of the equations.

$$
2 \mathrm{H}_{2} \mathrm{O} \rightarrow 2 \mathrm{H}_{2}+\mathrm{O}_{2}
$$

She was the equation written, but as we did laboratory tests, we found that apart from the elevated oxygen levels, also had flow of electrons; which was easy to explain because the reaction is reversible in appropriate conditions. So, eventually we perfect the reaction in the following manner:

$$
2 \mathrm{H}_{2} \mathrm{O} \rightarrow 2 \mathrm{H}_{2}+\mathrm{O}_{2} \rightarrow 2 \mathrm{H}_{2} \mathrm{O}+4 \mathrm{e}^{-}
$$

Melanin releases energy in the form of symmetrical in all directions. Something like increased energy spheres (Figure 8).

The position of the granules of melanin into the cell eucarionte is strategic since it is located mainly in the perinuclear space, allowing him to be the source of energy of the nucleus, which does not possess neither mitochondria nor ATP; and at the same time it floods the cell cytoplasm with these growing spheres of energy, which are distributed throughout the entire cell, following the laws of simple diffusion (Figure 9).

The process of transformation of light energy into chemical energy seems to be the same in all living organisms, including insects (Figure 10).

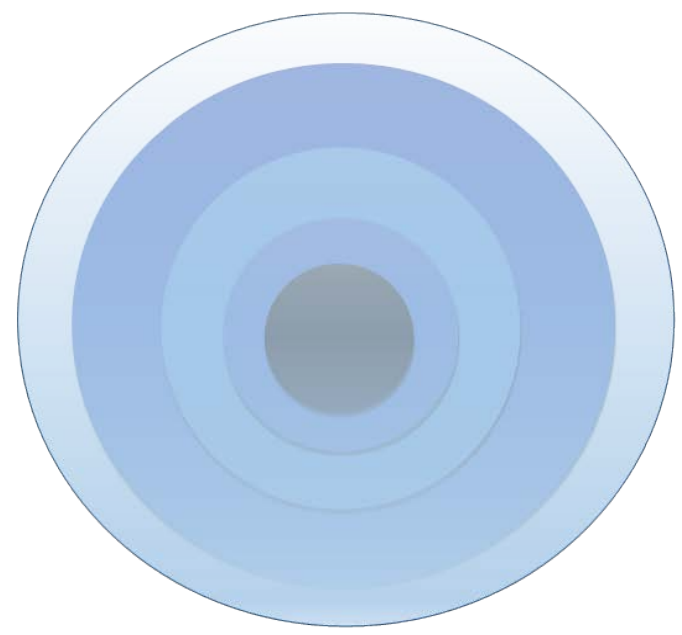

Figure 8. Melanin drawn to the Center, and the areas surrounding it, mean the different concentrations of $\mathrm{H}_{2}$ and $\mathrm{O}_{2}$ resulting of the dissociation of water, also the different concentrations of water reformed, and at the same time, also with their corresponding 4 highenergy electrons for every two molecules of re-formed water. 


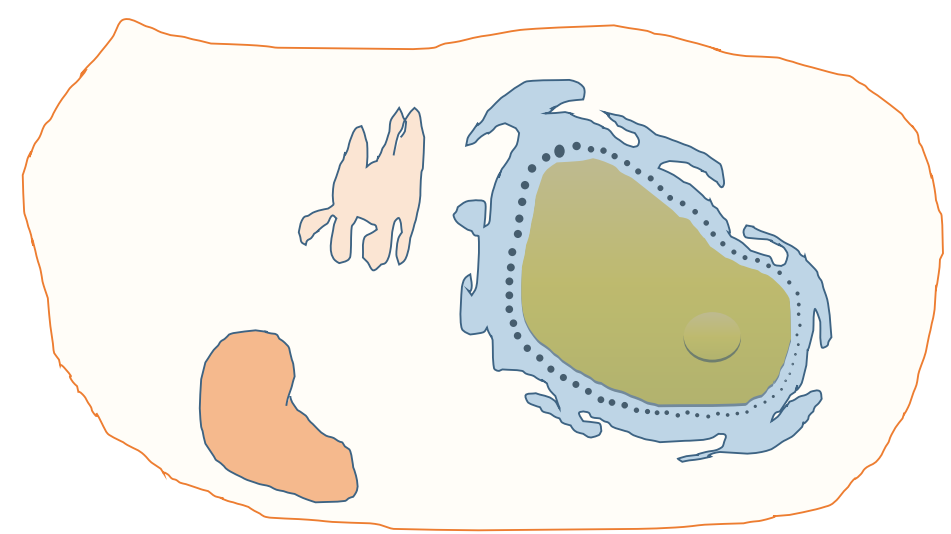

Figure 9. The scheme represents the cell membrane, mitochondria, Golgi apparatus, rough endoplasmic reticulum, which, like the granules of melanin (melanosomes) surrounds completely to the cell nucleus.

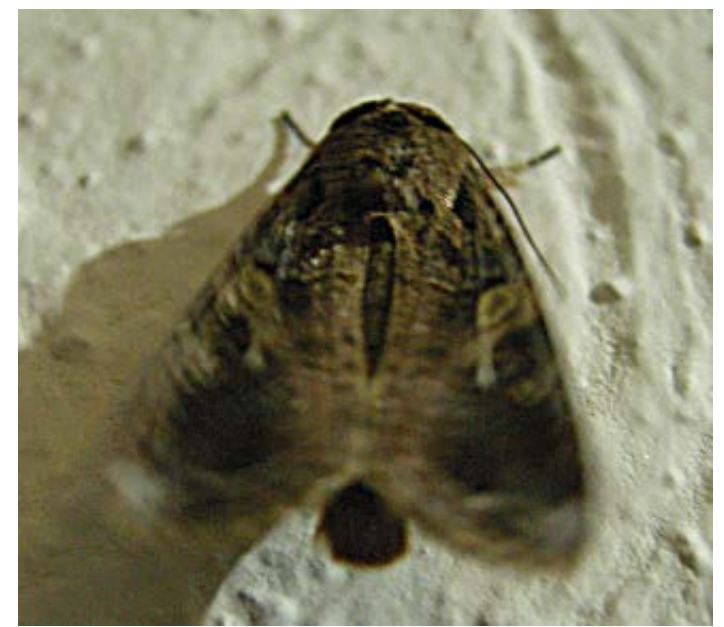

Figure 10. Melanin is also present in the common moth, and its main function is also transform light energy into chemical energy through dissociation and re-forming of the water molecule.

\section{Melanin and Plants}

The downward movement of sugary plant fluids in phloem requieres energy, indeed, and melanin is the explanation. Mistery about how plants move fluids long distances in their phloem is easiest of understand taking in account the energy that comes from melanin.

That sucrose is either used as an energy source for its own metabolism is an idea very difficult to explain. So far, there are only hypothesis, in example, hypothesis of how water rises up in the xylem to the tops of tall trees, how sucrose and a proton $\left(\mathrm{H}^{*}\right)$ are pumped into the cell; how water comes and build a pressure, how the sap can move a very significant distance; how sucrose is pumped out, how water is pumped out, how sucrose can be splitted to its monomers and thence to other hexoses.

Let us remember that any chemical reaction involves exchange of energy. The metabolism of plants as animals is poorly understood, since the power source was not known. Glucose is a special molecule that organisms use as a source of biomass as a source of carbon chains, but even there, and both animals and plants take the necessary energy from water. Energy can be defined as everything that produces a change, and the word metabolism means continuous change.

The metabolic needs of the plant are continuous, incessant, both day and night; and it is the way in which melanin delivered energy, in the form of incessant, continuous, both day and night. Therefore, the mysteries about life in the plants will have to look in the trunk, not so much in the leaves (Figure 11). 


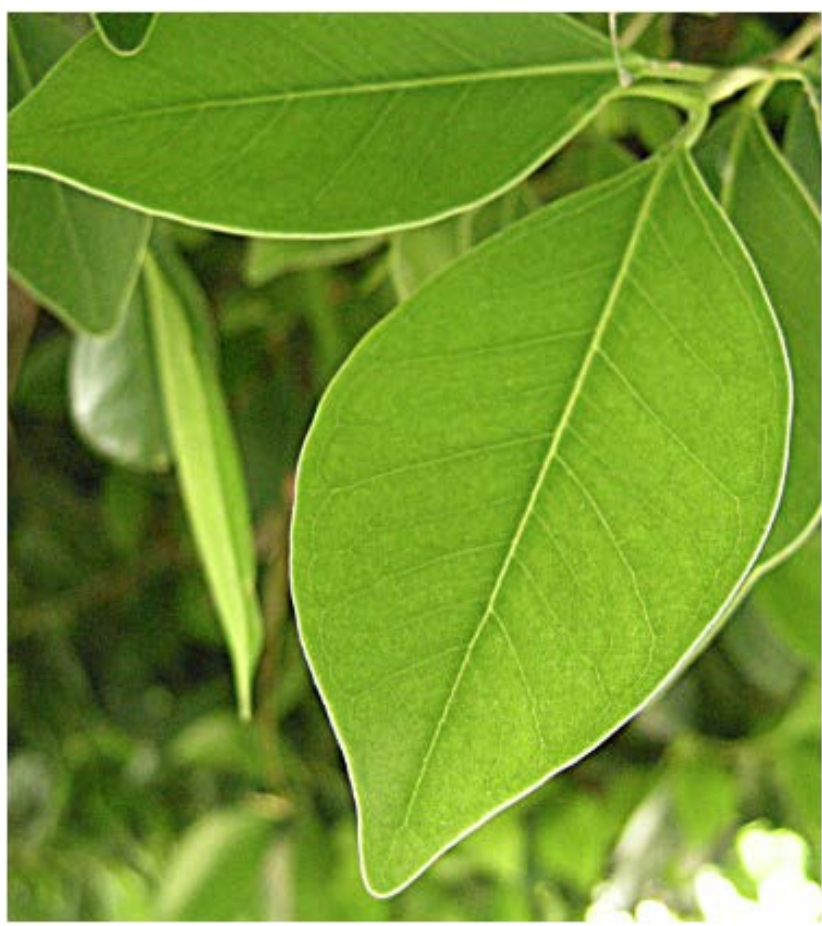

Figure 11. The chlorophyll in plants, as well as the hemoglobin in humans, possess the ability to dissociate the water molecule in an irreversible manner, using the light energy it contains. But both molecules are only an extension of the master molecule that is melanin [5].

\section{Conclusions}

Both plants and humans are enveloped in a dense cloud of mysteries, at least in regards to your metabolism. It is no coincidence that of the 7000 cellular reactions described in the literature, only about 200 are described in the same way in the different sources of information, there are significant disputes in the remaining 6800. And it is that cell biology has puzzled trying to concatenate the glucose as a source of energy and at the same time of carbon chains; which is to say that a wonderful molecule not only brings carbon atoms to organisms but also carries the necessary energy for its own metabolism.

This wrong concept is deeply rooted in both the scientist and public in general. But glucose just provides the building-blocks of the $99 \%$ of the biomolecules that mold our organism. Thereby the body knows glucose very well, so is able to split it, to combine it with other elements or molecules, and in a very precise way; even more, the cell is able to metabolize glucose to $\mathrm{CO}_{2}$ and water; but the energy that supposedly glucose contains is a myth. Therefore, that biology at the molecular level has been stagnant. It is not possible to extract more energy from glucose containing that.

However, if from now forward we take into account the unexpected role of melanin as an important source bioenergetics, the role of the glucose will refine and allows us to re-order our concepts in a manner more consistent with nature, where everything depends on the generation and distribution of energy.

The discovery that the main source of energy comes from lignin [6] and chlorophyll, represents a substantial change in the study of photosynthesis in plants. We will have to reconsider many metabolic pathways so far accepted dogmatically but remain without plausible explanation, whereas to date theories. A significant advance in the knowledge will be able to be here, and it is a sharp change of direction to redirect the attention toward the trunk of the plants, rather than the leaves; but this will allow us to better understand the mysteries of the physiology of plants.

\section{References}

[1] Hoffman, J. and Puszynski, A. (2009) Chemical Engineeering and Chemical Process Technology. Vol. V. Pigments 
and Dyestuffs. Encyclopedia of Life Support Systems (EOLSS).

[2] Solis Herrera, A., Arias Esparza, M.C., Solís Arias, R.I., et al. (2010) The Unexpected Capacity of Melanin to Dissociate the Water Molecule Fill the Gap between the Life before and after ATP. Biomedical Research, 21, 224-227.

[3] Anderson, A.N., Tobimatsu, Y., Ciesielki, P.N., Ximenes, E., Ralph, J., Donohoe, B.S., Ladisch, M. and Chapple, C. (2015) Manipulation of Guaiacil and Syringyl Monomer Byosynthesis in an Arabidopsis Cinnamyl Alcohol Dehydrogenase Mutant Results in Atypical Lignin Byosynthesis and Modified Cell Wall Structure. The Plant Cell, 27, 21952209. http://dx.doi.org/10.1105/tpc.15.00373

[4] Hill, H.Z. (1992) The Function of Melanin or Six Blind People Examine an Elephant. BioEssays, 14, 49-56. http://dx.doi.org/10.1002/bies.950140111

[5] Solís-Herrera, A. And Solís-Arias, M.P. (2013) The Odyssey of Atmospheric Oxygen in Their Futile Attempt to Reach the Interior of the Cell. International Journal of Health Research and Innovation, 1, 37-52.

[6] Solís-Herrera, A. And Arias-Esparza, M.C. and Solís Arias, M.P. (2015) Photosynthesis in Humans, Chapter 12 of the Book: Photosynthesis, Functional Genomics, Physiological Processes and Environmental Issues. Edited by Nafees Khan. Nova Publishers, New York, 257-279. 